04

\title{
Геометрооптический механизм восстановления волнового фронта
}

\author{
(C) А.М. Смолович \\ Институт радиотехники и электроники (ИРЭ) им. В.А. Котельникова РАН, \\ 125009 Москва, Россия \\ e-mail: asmolovich@petersmol.ru
}

Поступила в редакцию 10.12.2019 г.

В окончательной редакции 27.04.2020 г.

Принята к публикации 04.05.2020 г.

\begin{abstract}
Проанализирован механизм восстановления волнового фронта, основанный на геометрооптическом отражении восстанавливающего излучения от поверхностей постоянной разности фаз между объектной и опорной волнами, использованными для записи интерференционной картины в регистрирующей среде. Этот механизм сравнивается с голографическим. Проведены экспериментальные исследования в объемной регистрирующей среде и в планарном оптическом волноводе. Предложено несколько типов ахроматических оптических элементов. Обсужден оптико-акустический фокусирующий элемент, способный создавать большое локальное напряжение в области специальной формы внутри среды. Показана возможность восстановления временно́й структуры световых импульсов.
\end{abstract}

Ключевые слова: объемная голограмма, киноформ, ультракороткий импульс, временна́я форма импульса.

DOI: $10.21883 /$ OS.2020.09.49866.338-20

\section{Введение}

Механизм восстановления волнового фронта голограммой основан на дифракции восстанавливающего излучения записанной в голограмме квазипериодической интерференционной структурой. Именно в локальном периоде этой структуры заключена информация о волновом фронте объектной волны. Этот механизм работает как в тонких голограммах, так и в объемных, зарегистрированных как в сходящихся, так и во встречных пучках. Согласно другому механизму, предложенному в [1], волновой фронт объектной волны восстанавливается благодаря геометрооптическому (ГО) отражению восстанавливающего излучения от одиночной поверхности максимума интерференционной картины, которая действует подобно зеркалу переменной кривизны. Эта локальная кривизна и содержит информацию об объектном волновом фронте. В отличие от голографического восстановления при ГО восстановлении нет дифракции восстанавливающего излучения периодической интерференционной структурой. Это делает процесс восстановления ахроматическим, т.е. позволяет восстанавливать неискаженный волновой фронт светом с длиной волны, отличной от длины волны в процессе записи [2,3]. ГО механизм восстановления волнового фронта был предложен для голографии. Однако он существенно отличается от голографического и представляет самостоятельный интерес.

В работе механизм ГО восстановления волнового фронта сравнивается с голографическим. Мы объясняем, почему этот механизм не работает в обычных голограммах, в том числе в голограммах Денисюка. Для ГО восстановления волнового фронта используется запись в объемной регистрирующей среде с помощью ультракоротких лазерных импульсов. В другом варианте двумерный аналог эффекта получен в планарном оптическом волноводе. Предложено несколько типов ахроматических оптических и оптико-акустических элементов (ОЭ) и систем. Кроме того, обсуждается восстановление временно́й структуры ультракоротких световых импульсов.

\section{Принципы ГО восстановления}

Пусть объектная $A_{O}(\mathbf{r}) \exp \left[i k L_{O}(\mathbf{r})\right] \quad$ и $\quad$ опорная $A_{R}(\mathbf{r}) \exp \left[i k L_{R}(\mathbf{r})\right] \quad$ волны удовлетворяют скалярным уравнениям ГО ([4], параграф 1). Здесь $k=2 \pi / \lambda-$ волновое число и $\lambda$ - длина волны излучения, $L_{O}(\mathbf{r})$ и $L_{R}(\mathbf{r})$ - эйконалы волн, $A_{O}(\mathbf{r})$ и $A_{R}(\mathbf{r})-$ их амплитудные функции, $\mathbf{r}$ - радиус-вектор. Интенсивность интерференционного поля этих волн определяется выражением:

$$
A_{O}^{2}(\mathbf{r})+A_{R}^{2}(\mathbf{r})+2 A_{O}(\mathbf{r}) A_{R}(\mathbf{r}) \cos \left\{k\left[L_{R}(\mathbf{r})-L_{O}(\mathbf{r})\right]\right\} .
$$

Сначала рассмотрим случай, когда записывается тонкая голограмма (рис. 1,a). Пусть голограмма восстанавливается волной $A_{R}(\mathbf{r}) \exp \left[i k^{\prime} L_{R}(\mathbf{r})\right]$, где волновое число
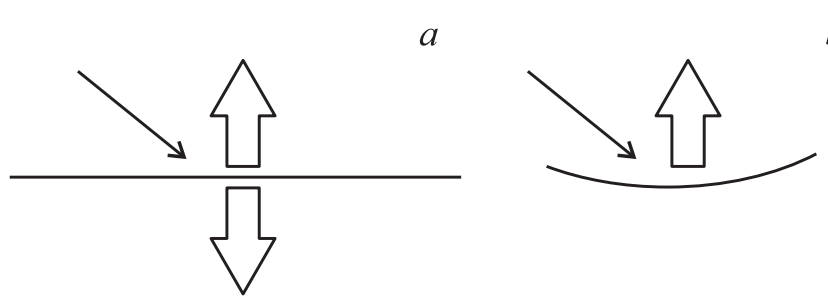

Рис. 1. Восстановление волнового фронта: $a-$ голограммой, $b-$ с помощью ГО механизма. 
$k^{\prime}=2 \pi / \lambda^{\prime}$ может отличаться от волнового числа при записи голограммы. Результирующее поле, полученное в результате взаимодействия (прохождения или отражения) восстанавливающей волны с голограммой, может быть получено из (1) в виде суммы, в которой слагаемое, связанное с восстановленным волновым фронтом (ВФ), пропорционально [5]:

$$
A_{O}(\mathbf{r}) A_{R}^{2} \exp \left\{i\left[\left(k^{\prime}-k\right) L_{R}(\mathbf{r})+k L_{O}(\mathbf{r})\right]\right\} .
$$

Волна (2) имеет эйконал:

$$
L_{d i f f r}(\mathbf{r})=\left(\lambda^{\prime} / \lambda\right) L_{O}(\mathbf{r})+\left(1-\lambda^{\prime} / \lambda\right) L_{R}(\mathbf{r}) .
$$

Отсюда следует, что эйконал объектной волны будет восстанавливаться только при $\lambda^{\prime}=\lambda$. При произвольном $\lambda^{\prime}$ выражение (3) описывает зависимость формы восстановленного ВФ от длины волны (дисперсию), и для случая плоских волн приводит к формуле решетки (секция 8.6.1, выражение (8) в [6]), а для сферических волн к формулам Меира [7].

Следуя [1], рассмотрим теперь поверхности, на которых аргумент косинуса в (1) будет постоянным (рис. $1, b)$. Для краткости будем их называть изофазными поверхностями, хотя более точно это поверхности, на которых разность фаз интерферирующих волн постоянна, или поверхности, на которых постоянна интенсивность суммарного поля. Эти поверхности определяются уравнением

$$
L_{R}(\mathbf{r})-L_{O}(\mathbf{r})=p,
$$

где $p$ является константой для каждой изофазной поверхности. Если восстанавливающая волна $A_{R}(\mathbf{r}) \exp \left[i k^{\prime} L_{R}(\mathbf{r})\right]$ испытывает ГО отражение от поверхности (4), то фаза $k^{\prime} L_{r e f l}(\mathbf{r})$ отраженной волны на этой поверхности совпадает с фазой восстанавливающей волны [4]:

$$
k^{\prime} L_{r e f l}(\mathbf{r})=k^{\prime} L_{R}(\mathbf{r})
$$

Из (4) и (5) получаем

$$
L_{r e f l}(\mathbf{r})=L_{O}(\mathbf{r})+p,
$$

т.е. с точностью до аддитивной константы эйконал объектной волны восстанавливается при любом значении $k^{\prime}$ в отличие от случая тонкой голограммы. Из выражения (6) также следует, что волновой фронт объектной волны (поверхность $L_{O}(\mathbf{r})=$ const) восстанавливается ахроматически. Таким образом, волны, восстановленные с помощью этих двух механизмов, не совпадают при $\lambda^{\prime} \neq \lambda$.

Следующие простые примеры демонстрируют разницу между голографическим восстановлением и ГО восстановлением. В простейшем случае плоских объектной и опорной волн голографический механизм соответствует дифракции плоской волны решеткой, а ГО механизм соответствует отражению восстанавливающей волны плоским зеркалом. Если объектная волна сферическая, соответствующими структурами являются зонная пластинка
Френеля и параболическое зеркало. В общем случае ГО восстановление подобно отражению от зеркальной поверхности сложной кривизны.

Обычная объемная голограмма содержит фрагменты поверхностей максимумов интерференционной картины. Они формируют периодическую структуру, содержащую большое количество таких поверхностей. Как правило, объемная голограмма регистрируется в плоскопараллельном слое толщиной от нескольких микрон до нескольких десятков микрон, имеющем поперечные размеры от нескольких сантиметров до нескольких десятков сантиметров. Таким образом, протяженность периодической структуры вдоль поверхности голограммы обычно на несколько порядков превышает размеры каждого фрагмента поверхности интерференционного максимума внутри слоя (рис. 2,a). Вследствие этого дифракционный механизм восстановления доминирует над ГО механизмом $[8,9]$. Это подтверждается наличием зависимости направления восстановленного объемной голограммой пучка от длины волны восстанавливающего излучения, которую можно наблюдать в пределах спектральной селективности голограммы.

\section{Эксперименты}

Чтобы получить ГО восстановление, количество максимумов интерференционной картины, зарегистрированных в объеме среды, должно быть уменьшено, а толщина регистрирующей среды увеличена. Для этого может быть использована регистрация интерференции ультракоротких импульсов лазерного излучения $[10,11]$. Условие доминирования ГО механизма для голограммы, зарегистрированной в плоскопараллельном слое, определяется следующим критерием [9]:

$$
T \cos \psi \gg D
$$

где $T$ - толщина плоскопараллельного слоя, $\psi-$ угол между вектором решетки и нормалью к поверхности слоя, $D$ - ширина зарегистрированной интерференционной структуры в направлении, нормальном к изофазным плоскостям (рис. 2,b). В случае, если голограмма записана двумя короткими импульсами, которые имеют одинаковую длительность $\tau$, и распространяются прямо навстречу друг другу, $D \sim \tau c$, где $c-$ скорость света в регистрирующей среде. Критерий (7) был получен в [8,9] с помощью классического подхода Габора-Строука [12].

Для регистрации использовался лазер фемтосекундных импульсов на титанате сапфира с длиной волны излучения, варьируемой в диапазоне 780-830 nm. Длительность импульсов была порядка $40 \mathrm{fs}$. Частота повторения импульсов была равна $80 \mathrm{MHz}$, а средняя мощность излучения - $200 \mathrm{~mW}$. Лазерный пучок делился с помощью полупрозрачного интерференционного зеркала на два пучка, которые направлялись навстречу друг другу. Регистрирующая пластинка устанавливалась 

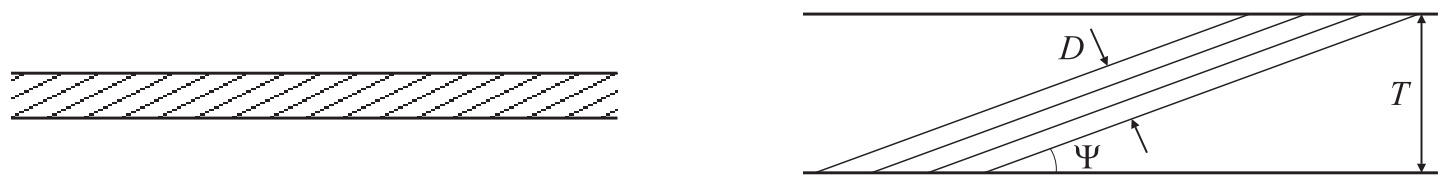

Рис. 2. Поверхности максимумов интерференционной картины: $a-$ в обычной объемной голограмме, $b-$ в структуре, зарегистрированной ультракороткими импульсами в материале увеличенной толщины.

так, чтобы ее фотослой находился в области перекрытия встречных импульсов. Для точного уравнивания длин оптических путей использовалась методика, предложенная в [13]. Нормаль к пластинке составляла угол порядка $20^{\circ}$ с оптической осью. Использовались специально изготовленные фотопластинки с толщиной фотослоя от 110 до $280 \mu \mathrm{m}$. Экспозиция подбиралась экспериментально и имела порядок нескольких десятых джоуля на квадратный сантиметр. На каждой фотопластинке засвечивалось несколько полей с разной экспозицией. Для контроля некоторые поля экспонировались непрерывным излучением в той же геометрии, что и импульсным. Восстановление производилось в отражательной геометрии. Для восстановления использовался лазер на красителе (раствор родамина 6G в этиленгликоле) с накачкой аргоновым лазером. Использовался непрерывный режим генерации с перестройкой длины волны излучения от 580 до $630 \mathrm{~nm}$. Восстановленный пучок света наблюдался на экране, установленном на расстоянии порядка $4 \mathrm{~m}$ от фотопластинки. Восстановленный пучок мог наблюдаться при изменении длины волны лазера от 585 до $607 \mathrm{~nm}$. При этом изменении пятно, образованное на экране восстановленным пучком, не смещалось. В противоположность этому пятно от пучка, восстановленного голограммой, зарегистрированной непрерывным излучением в тех же условиях, смещалось на $6 \mathrm{~cm}$. Из этого мы можем сделать вывод, что для структуры, зарегистрированной фемтосекундными импульсами, работает ГО механизм восстановления.

Однако большая толщина регистрирующей среды, которая требуется для наблюдения эффекта, является серьезным препятствием для его применения. Фотографическая обработка среды, имеющей такую большую толщину, вызывает очевидные технические трудности. Кроме того, в таких слоях поглощение света во время регистрации [14] приводит к существенному снижению дифракционной эффективности $[15,16]$. Для преодоления этих проблем мы предлагаем использовать волноводный аналог эффекта ГО восстановления. Предположим, что голограмма регистрируется в планарном волноводе ультракороткими лазерными импульсами, которые проникают в волновод через его верхнюю границу (рис. 3,a). Восстановление происходит в волноводном режиме (рис. 3,b). В этом случае процесс восстановления является двумерным. Вид сверху на волноводную голограмму, на которую падает восстанавливающий пучок света, будет подобен поперечному сечению в трехмерном случае (рис. 2,b). Поэтому мы можем использовать критерий (7) доминирования механизма ГО восстановления, заменяя в нем толщину слоя $T$ на ширину восстанавливающего пучка в плоскости волновода. Здесь ширина структуры $D \sim \tau c / \sin (\varphi / 2)$, где $\varphi-$ угол между пучками, записывающими голограмму. Отсюда можно понять, что в этом случае условия ГО восстановления не зависят от толщины планарного волновода. Это является главным преимуществом волноводного ГО восстановления над его трехмерным аналогом.

В эксперименте в качестве модели планарного волновода использовался слой бихромированной желатины $\left(n_{2}=1.54\right)$ толщиной $20 \mu \mathrm{m}$ на подложке из полированного кварца $\left(n_{1}=1.456\right)$. Для регистрации использовалась вторая гармоника $(\lambda=400 \mathrm{~nm})$ лазера на титанате сапфира. Длительность импульсов была порядка $30 \mathrm{fs}$. Bo время регистрации импульсы расщеплялись и направлялись на волноводный образец через его верхнюю поверхность (рис. 3,a). Угол $\varphi$ между записывающими пучками был около $60^{\circ}$. Обработка бихромированной желатины после экспозиции включала промывку в холодной воде и сушку в растворах изопропилового спирта возрастающей концентрации. Восстановление производилось в волноводном режиме (рис. $3, b$ ). Для ввода/вывода излучения в планарный волновод использовались призмы из алюмоиттриевого граната с углом $60^{\circ}$ у основания. Для восстановления использовался лазер на красителе (раствор родамина 6G в этиленгликоле) с накачкой

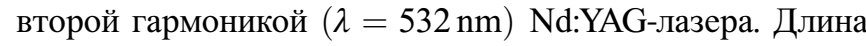
волны восстанавливающего излучения варьировала от 580 до $630 \mathrm{~nm}$. Восстановленный пучок после вывода из волновода наблюдался в пределах спектральной селективности голограммы в диапазоне 580-615 nm. Направление восстановленного пучка определялось с точностью $6 \cdot 10^{-4} \mathrm{rad}$. При голографическом восстановлении оно должно было бы зависеть от длины волны. В этом случае изменение расчетного значения угла $\alpha$ в пределах спектральной селективности структуры составляло бы порядка $0.03 \mathrm{rad}$. Измеренное в эксперименте значение угла $\alpha$ не менялось. Это доказывает, что в данном случае работает ГО механизм восстановления волнового фронта.

Вместо непосредственной голографической записи возможен цифровой синтез волноводной ахроматической голограммы, содержащей всего несколько пери- 

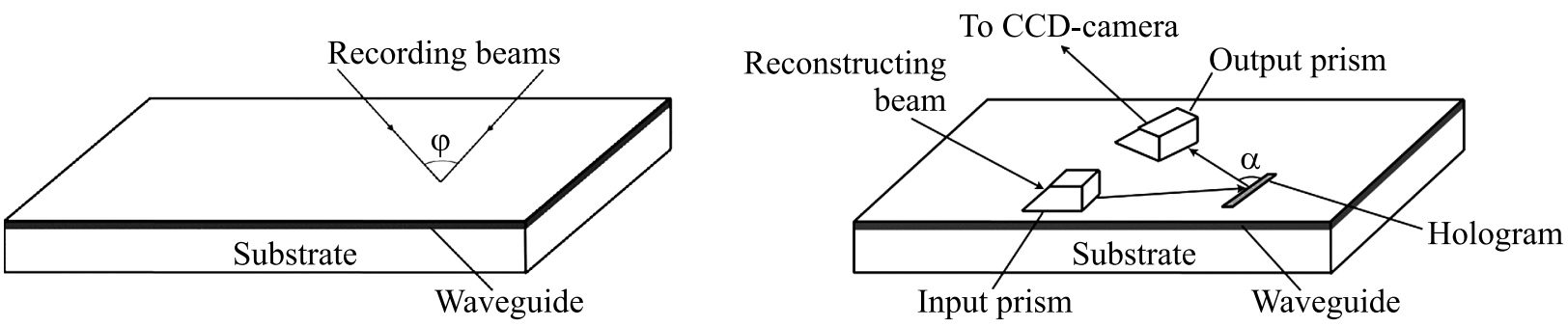

Рис. 3. ГО механизм в планарном волноводе: $a-$ регистрация, $b-$ восстановление.

одов. Мы предлагаем для изготовления волноводных ОЭ использовать нанотехнологии с фокусированным ионным пучком (FIB). В [16] была продемонстрирована возможность получения этим способом периодической структуры с большим аспектным отношением. Мы использовали установку Strata $^{\text {TM }}$ FIB201 с галлиевым ионным пучком, минимальный диаметр которого был равен $7 \mathrm{~nm}$. Использовались два варианта. В первом периодическая структура непосредственно формировалась на слое полимера. Во втором периодическая структура сначала формировалась на кремниевой пластине, а затем реплицировалась на полимере ультрафиолетового отверждения. Полимерные образцы были изготовлены В.И. Соколовым (Институт проблем лазерных и информационных технологий РАН, Троицк, Московская обл., Россия). Периодические структуры, сформированные на полимере и кремнии, показаны на рис. 4, $a$ и $b$ соответственно. Фотографии были получены на FIB во вторичных электронах. Чтобы увидеть профиль решетки, в образце вытравливалось специальное прямоугольное углубление, нормальное к штрихам решетки. Фотографии сделаны при наклоне образца на $45^{\circ}$. Были получены периодические структуры с отношением глубины штриха к его ширине порядка 20. Ширина штриха вблизи вершины составляла $200 \mathrm{~nm}$.

Ахроматические волноводные ОЭ могут использоваться для оперирования ультракороткими лазерными импульсами, которые имеют широкий спектр. Кроме того, они могут использоваться для параллельной обработки сигналов на различных частотах. Оба направления нужны для повышения скорости обработки информации. Возможные функции подобных элементов включают фокусировку пучков, ввод/вывод пучков, связь между планарным и волоконным волноводами.

\section{Ахроматические оптические и оптико-акустические элементы и системы}

Как и в двумерном случае, трехмерные ахроматические ОЭ могут быть получены не прямой регистрацией, а с использованием методов цифровой голографии, когда сначала рассчитывается форма ОЭ, a затем он синтезируется тем или иным способом. В [17] Стетсон обсуждал принципиальную возможность ахроматического восстановления волнового фронта гипотетической изолированной изофазной поверхностью, если ее сделать бесконечно тонкой и зеркально отражающей. Эта научно-популярная статья была очень важна благодаря последовавшим за ней работам. В [18] Шеридон, сославшись на статью Стетсона [17], обратил внимание на то, что соседние поверхности максимумов интерференционной картины имеют очень близкую форму. Этот факт был использован для следующего эксперимента. Шеридон регистрировал на фоторезисте голограмму в отражательной геометрии. После обработки фоторезиста его поверхность приобрела форму, подобную решетке с пилообразным (blazed) рельефом. Каждый штрих этой решетки являлся частью некоторой поверхности максимумов интерференционной картины. Соседние штрихи относились к соседним поверхностям максимумов. Скачок высоты рельефа между штрихами соответствовал скачку фазы на $2 \pi$. Годом позже был предложен синтезированный на компьютере ОЭ, названный киноформом [19], который был подобен пилообразной голограмме Шеридона [18]. Позднее были разработаны так называемые „глубокие“ киноформы, называемые также multiorder или harmonic дифракционными линзами, в которых скачок фазы на границе зон равнялся $2 \pi N$ (где $N$ - натуральное число) [20-24]. Однако голограммы с пилообразным рельефом и киноформы не ахроматичны. Очевидно, что скачки поверхностного рельефа могут соответствовать $2 \pi$ или $2 \pi N$ скачкам фазы только для определенной длины волны излучения. Чтобы сделать ОЭ ахроматичным, мы должны исключить скачки фазы. ОЭ, ахроматически восстанавливающий некоторый волновой фронт, должен быть оптически эквивалентен одной из поверхностей максимумов интерференционной картины, когда волна, имеющая этот волновой фронт, интерферирует с какойто опорной волной. Форма этой поверхности должна быть рассчитана или измерена экспериментально. Синтезированный ОЭ может быть отражательным или пропускающим. Форма поверхности отражательного ОЭ в простейшем случае просто совпадает с формой поверхности максимумов интерференционной картины. При 
$a$
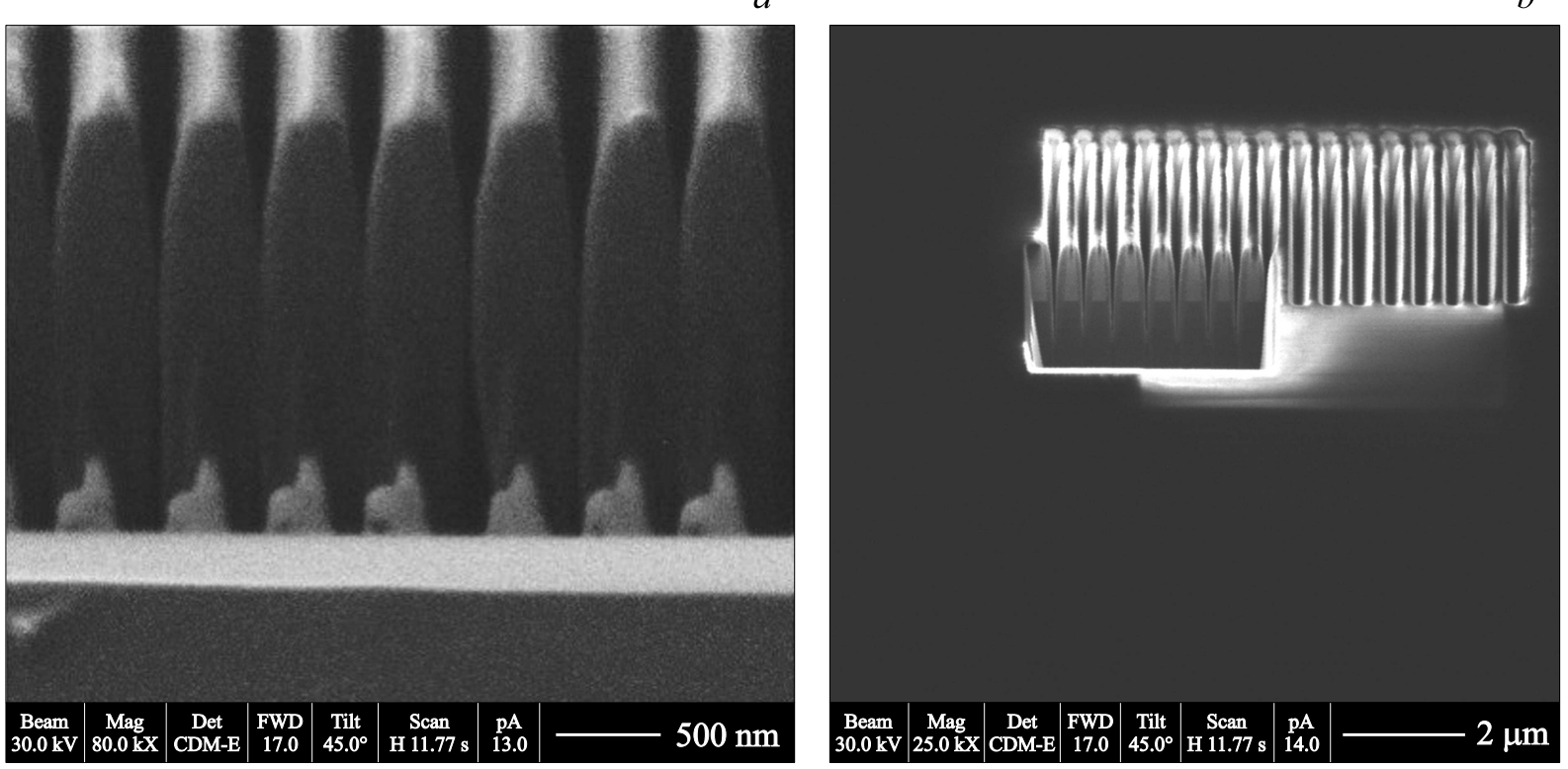

Pис. 4. Изготовление решетки с помощью FIB: $a-$ на полимере, $b-$ на кремнии.

переходе от отражательного ОЭ к его пропускающему эквиваленту координата каждой точки поверхности ОЭ вдоль его оптической оси должна быть умножена на $2 /(n-1)$ (где $n-$ показатель преломления материала, из которого изготовлен пропускающий ОЭ). Технологии получения ОЭ с поверхностью необходимой формы зависят от этой формы, размеров ОЭ и необходимой точности [3]. В случае, если возможности технологии не позволяют получить требуемый диапазон изменения высоты поверхности ОЭ, может быть использован какойто из вариантов, описанных ниже.

В первом варианте предлагается компенсация скачка фазы $[3,25]$. Оптическая система, показанная на рис. 5, $a$, содержит ступенчатый ОЭ (SE) и ОЭ с пилообразным рельефом (BE), которые расположены близко друг к другу. Подобно киноформу [22] поверхность ВЕ разделена на зоны. Высота рельефа меняется плавно в пределах каждой зоны и испытывает скачок на границе между соседними зонами. Однако в отличие от киноформа здесь величина скачка не связана с длиной волны излучения. Рабочая поверхность каждой ступени SE представляет собой участок плоской поверхности, нормальной к оптической оси. Каждая ступень SE имеет соответствующую ей зону ВЕ. Они расположены напротив друг друга и имеют одинаковую проекцию на плоскость, нормальную оптической оси. Разница в оптической длине пути между соседними ступенями SE равна по абсолютной величине и противоположна по знаку скачку на границе соответствующих зон ВЕ.

Рассмотрим, как работает оптическая система, изображенная на рис. 5, $a$. Пренебрежем рефракцией в оптической системе (не будем рассматривать наклонные лучи). Кроме того, предположим, что для всех зон $l_{i} \ll D_{i}^{2} / \lambda$, где $l_{i}-$ среднее расстояние между $i$-й зоной $\mathrm{BE}$ и соответствующей ступенькой $\mathrm{SE}$, а $D_{i}-$ ширина зоны (и ступеньки). В первую очередь рассмотрим опцию, когда элемент ВЕ пропускающий, а $\mathrm{SE}$ - отражающий. Сначала излучение проходит через BE, приобретая некоторый набег фазы. На границе между зонами 1 и 2 (рис. 5, $a$ ) длина оптического пути скачком меняется на величину, равную $a\left(n_{1}-n_{0}\right)$, где $a$ - величина скачка рельефа на границе зон, $n_{1}$ - показатель преломления материала $\mathrm{BE}$ и $n_{0}-$ показатель преломления среды между BE и SE. Затем излучение отражается от поверхности SE. Оптическая разность хода между лучами, отраженными от ступенек 3 и 4, соответствующим зонам 1 и 2, равна $2 h n_{0}$, где $h$ - высота ступеньки 3. Затем излучение второй раз проходит через ВЕ. Мы предполагаем, что лучи, прошедшие через каждую зону ВЕ, будут отражаться только ступенькой SE, соответствующей этой зоне, и наоборот. Тогда общая оптическая разность хода между лучами, прошедшими через оптическую систему через зоны 1 и 2 вблизи границы между ними, будет равна $2 a\left(n_{1}-n_{0}\right)-2 h n_{0}$. В этом случае условие компенсации скачков фазы, упомянутых выше, может быть записано как:

$$
h n_{0}=a\left(n_{1}-n_{0}\right)
$$

Оптическая система, для которой выполняется соотношение (8), эквивалентна ОЭ с непрерывным изменением высоты рельефа. Если пренебречь дисперсией показателя преломления материала, такая оптическая система может рассматриваться как ахроматическая. Заметим, что дисперсия показателя преломления значительно ниже дисперсии, связанной с дифракцией излучения на периодической структуре дифракционного ОЭ [20]. 

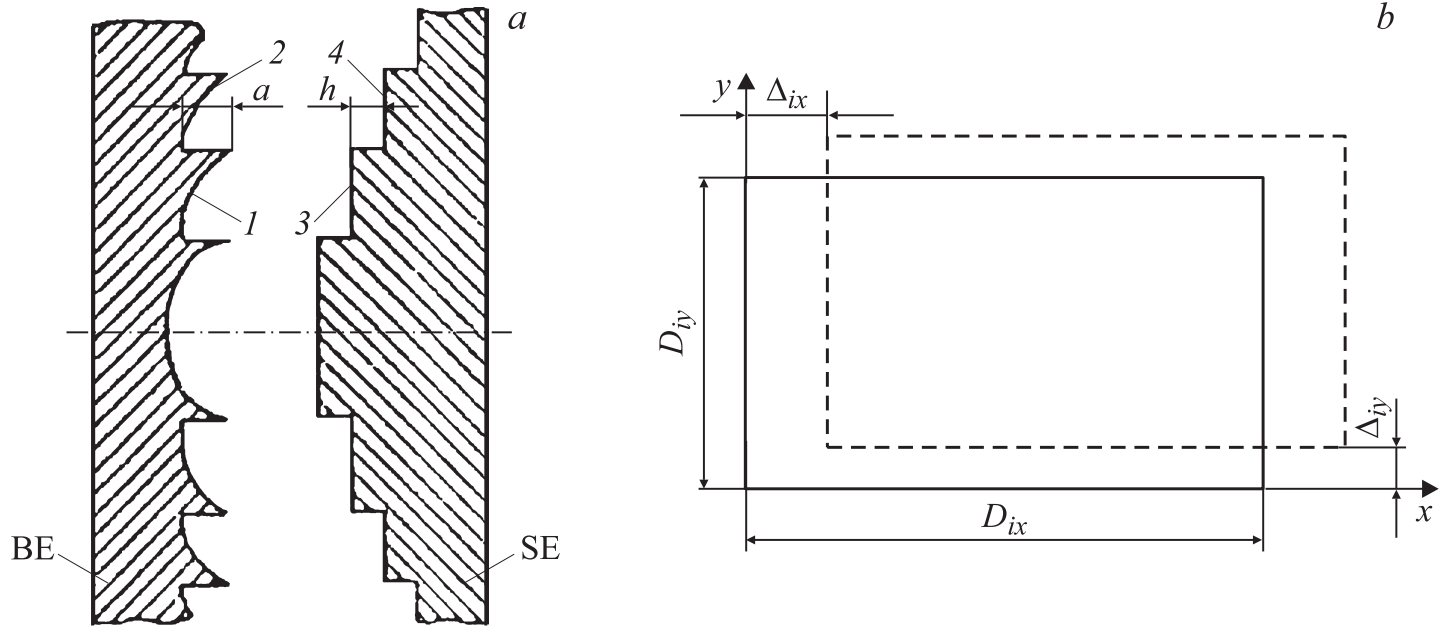

Рис. 5. $a$ - ахроматическая оптическая система, содержащая ОЭ с пилообразным рельефом (BE) и ступенчатый ОЭ (SE) (1,2 зоны $\mathrm{BE}, 3,4$ - ступеньки $\mathrm{SE}) . b$ - сдвиг лучевой проекции $i$-й зоны $\mathrm{BE}$ относительно соответствующей ей ступеньки $\mathrm{SE}$.

В другой опции в оптической системе ВE - отражающий, а SE - пропускающий. В этом случае условие компенсации скачков фазы будет следующим:

$$
h\left(n_{2}-n_{0}\right)=a n_{0},
$$

$n_{2}$ - показатель преломления материала SE.

В третьей опции оба элемента оптической системы являются пропускающими. В этом случае условие компенсации скачков фазы будет следующим:

$$
h\left(n_{2}-n_{0}\right)=a\left(n_{1}-n_{0}\right) .
$$

В случае $n_{1}=n_{2}, h=a$. BE и SE могут быть выполнены на противоположных сторонах одной и той же подложки.

До сих пор мы не рассматривали наклонные пучки в оптической системе (8)-(10). Сейчас оценим их вклад. Сначала рассмотрим случай, когда оба ОЭ являются пропускающими. Из-за наклона лучей часть излучения из некоторой зоны ВE не попадет на соответствующую ей ступеньку SE. Будем рассматривать эту часть излучения как шум. Тогда часть излучения, попавшая на соответствующую ступеньку, рассматривается как сигнал. Пусть ось $z$ направлена вдоль оптической оси, и $\theta-$ угол наклона лучей к оси $z$. $\theta_{x}$ и $\theta_{y}-$ проекции угла $\theta$ на плоскости $x z$ и $y z$ соответственно. Для быстрой оценки нижнего предела отношения сигнал/шум $(S / N)$ будем использовать модель, в которой все лучи из некоторой текущей $i$-й зоны имеют одинаковый угол наклона с проекциями $\theta_{i x}^{\max }$ и $\theta_{i y}^{\max }$. Предположим, что все зоны имеют прямоугольную форму и размеры $i$-й зоны равны $D_{i x}$ и $D_{i y}$ вдоль направлений соответствующих осей. Из-за наклона лучей лучевая проекция $i$-й зоны $\mathrm{BE}$ будет сдвинута по отношению к соответствующей ей ступеньки $\mathrm{SE}$ на $\Delta_{i x}$ и $\Delta_{i y}$ вдоль соответствующих осей (рис. $5, b$ ). Сдвинутая лучевая проекция текущей $i$-й зоны показана на рис. 5, $b$ штрихами, а соответствующая ей ступенька - сплошной линией. Сдвиги $\Delta_{i x}$ и $\Delta_{i y}$ равны:

$$
\begin{aligned}
& \Delta_{i x}=l_{i} \operatorname{tg}\left(\theta_{i x}^{\max }\right) \sim l_{i} \theta_{i x}^{\max }, \\
& \Delta_{i y}=l_{i} \operatorname{tg}\left(\theta_{i y}^{\max }\right) \sim l_{i} \theta_{i y}^{\max },
\end{aligned}
$$

где $l_{i}$ - среднее расстояние между поверхностями ОЭ. В (11) мы заменили тангенсы их аргументами. В нашей модели нижний предел отношения $S / N$ пропорционален отношению площадей сдвинутой проекции зоны, первая из которых внутри, а вторая снаружи соответствующей ступеньки (рис. 5, $b$ ):

$$
\begin{aligned}
S / N & >\sum_{i=1}^{N} \frac{\left(D_{i x}-\Delta_{i x}\right)\left(D_{i y}-\Delta_{i y}\right)}{D_{i x} D_{i y}-\left(D_{i x}-\Delta_{i x}\right)\left(D_{i y}-\Delta_{i y}\right)} \\
& =\sum_{i=1}^{N} \frac{\left(D_{i x}-l_{i} \theta_{i x}^{\max }\right)\left(D_{i y}-l_{i} \theta_{i y}^{\max }\right)}{D_{i x} D_{i y}-\left(D_{i x}-l_{i} \theta_{i x}^{\max }\right)\left(D_{i y}-l_{i} \theta_{i y}^{\max }\right)} \\
& \approx \sum_{i=1}^{N}\left(\frac{D_{i x} D_{i y}}{l_{i}\left(D_{i x} \theta_{i y}^{\max }+D_{i y} \theta_{i x}^{\max }\right)}-1\right) \\
& >\frac{1}{l \theta} \sum_{i=1}^{N} \frac{D_{i x} D_{i y}}{D_{i x}+D_{i y}}-N,
\end{aligned}
$$

где $N-$ количество зон, $l$ и $\theta-$ максимальные значения $l_{i}$ и $\theta_{i x, y}^{\max }$ соответственно. В (12) мы отбросили слагаемые, пропорциональные второй степени по $\theta_{i x, y}^{\max }$, рассматривая эту величину как малый параметр. Для случая, когда один из ОЭ отражательный, $l$ должно быть увеличено вдвое (пропорционально увеличению оптической длины пути для этого случая). Для некоторых специальных типов ОЭ (например, осесимметричных и т. п.) оценка отношения сигнал/шум может быть сделана точнее.

В [3] был предложен другой вариант ахроматической оптической системы, состоящей из двух ОЭ, не разделенных на зоны. Первый ОЭ имеет большую оптическую 


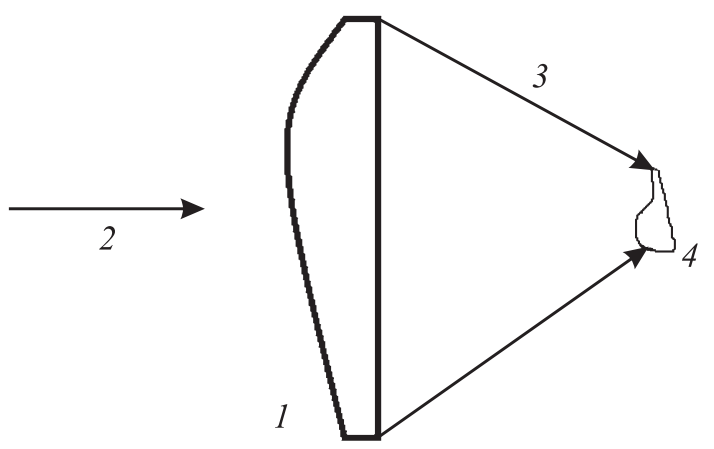

Pис. 6. Оптико-акустический фокусирующий элемент: 1 оптико-акустический элемент, 2 - лазерные импульсы, 3 акустические импульсы, 4 - область создания напряжений.

силу. Мы предполагаем, что его поверхность может иметь отклонения от расчетной формы, связанные с погрешностями изготовления. Эти погрешности компенсируются вторым ОЭ небольшой оптической силы. Второй ОЭ изготавливается по результатам измерений волнового фронта, сформированного первым ОЭ. Изготовить ОЭ с небольшой оптической силой гораздо проще. Опция, когда ОЭ с большой оптической силой отражающий, а с небольшой оптической силой пропускающий, здесь предпочтительна, поскольку в этом случае хроматические аберрации системы, связанные с дисперсией материала пропускающего ОЭ, должны быть минимальны.

В [26] был предложен ахроматический ОЭ, относящийся к классу ОЭ специального типа, именуемых фокусаторами. Согласно данной опции, разные зоны ОЭ фокусируют излучение в различные, не пересекающиеся между собой, части области фокусировки. Таким образом, каждая зона фокусатора работает, как независимый ГО ОЭ.

Фокусирующий оптико-акустический элемент, изображенный на рис. 6 [27], работает следующим образом. На поверхность элемента направляется лазерный импульс. Сходящаяся акустическая волна генерируется в результате быстрого поглощения света вблизи поверхности оптико-акустического элемента. Патент [27] базируется на экспериментальных результатах Г.А. Аскарьяна с сотрудниками $[28,29]$, в которых мощные акустические импульсы генерировались на сферической поверхности и фокусировались в точку. Используя мощные лазерные импульсы или последовательности импульсов, a также взрывающиеся под действием электрического разряда металлизированные пленки, Г.А. Аскарьян получил вблизи фокуса акустической волны давления более $10^{3} \mathrm{~atm}$. В [27] предлагается вместо сферической поверхности использовать элемент со сложной формой поверхности, которая рассчитывается путем решения обратной задачи для фокусировки акустической волны в область нужной конфигурации. Здесь использование ГО элемента является принципиальным, поскольку генерируемый акустический импульс может иметь лишь один период колебаний [29], т. е. его спектр будет очень широким. Поэтому дифракционные элементы здесь не подойдут. Требования к точности изготовления фокусирующего оптико-акустического элемента значительно ниже, чем в оптике из-за разницы в длинах волн. Такой элемент может быть изготовлен, например, на станке с ЧПУ. В [27] предлагается использовать эту технологию для торможения роста трещин в металлических изделиях за счет создания зоны пластической деформации на пути развития трещины.

Мы предполагаем, что эта технология может быть использована и в других областях, в том числе для проведения хирургических операций на внутренних органах без разрезания внешних тканей тела. Метод дает принципиальную возможность имитировать действия скальпеля или других хирургических инструментов. Для симуляции движения хирургических инструментов внутри тела возможны две опции. Согласно первой, используется движение оптико-акустического фокусирующего элемента вдоль тела пациента. Элемент должен быть соединен с телом через иммерсию. Во второй опции фокусирующий оптико-акустический элемент выполняется вытянутым вдоль пути движения скальпеля, а лазерный пучок движется вдоль элемента. Кроме того, мы предполагаем, что данная технология может применяться для симуляции действия бандажа при остановке внутренних кровотечений.

\section{Временно́е восстановление}

Другие эффекты возникают, когда ультракороткие лазерные импульсы используются не только для записи
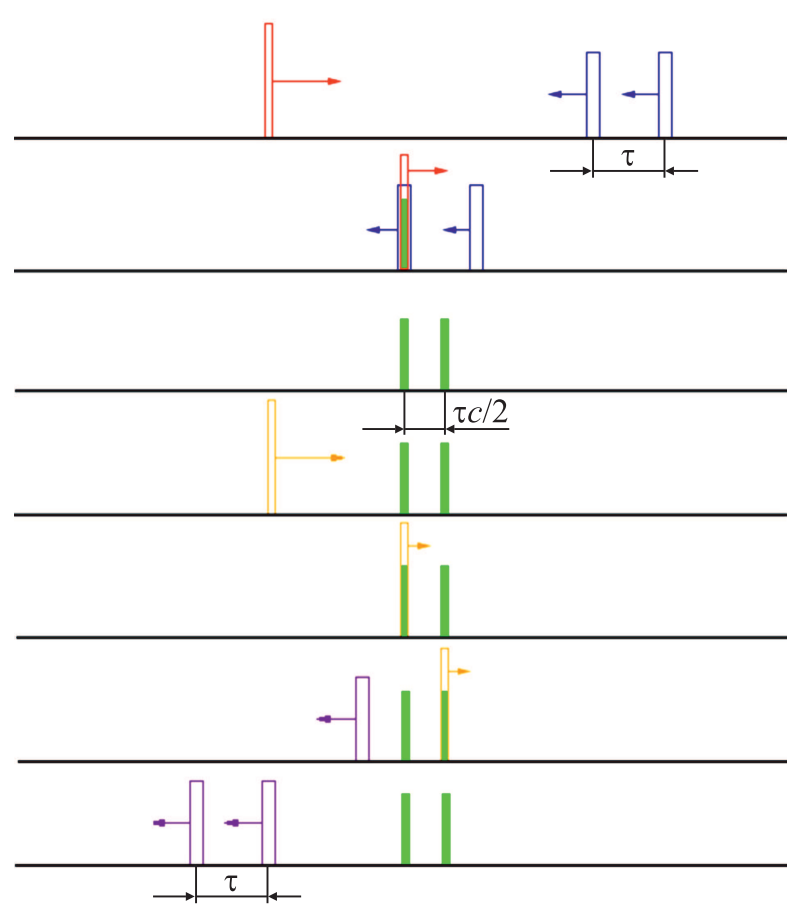

Рис. 7. Временно́е восстановление светового импульса. 
голограммы, но и при восстановлении [30]. Среди них восстановление временно́й структуры импульса. Следующий простой пример показывает, как это работает (рис. 7). Предположим, что длительность опорного импульса значительно меньше, чем длительность объектного сигнала. Поэтому мы будем рассматривать опорный импульс как $\delta$-импульс. Пусть объектный сигнал представляет собой два $\delta$-импульса, разделенных временным промежутком $\tau$. Предполагаем, что голограмма записывается в отражательной геометрии. Тогда область пространственно-временного пересечения объектного и опорного сигналов представляет собой две плоскости, перпендикулярные направлению распространения волн, отстоящие друг от друга на расстояние $\tau c / 2$, где $c-$ скорость света. Пусть в качестве восстанавливающей волны также используется $\delta$-импульс, который падает на зарегистрированную структуру с той же стороны, что и опорный импульс при регистрации. Он частично отражается сначала от первой плоскости, а затем от второй. Полученный в результате этого сигнал будет представлять собой два $\delta$-импульса, разделенных временным промежутком $\tau$. Таким образом, временная структура объектного импульса была восстановлена с помощью простого ГО отражения.

При использовании восстанавливающей волны, сопряженной с опорной, можно получить объектный сигнал, обращенный во времени. В нашем примере, показанном на рис. 7, это соответствует случаю, когда восстанавливающий $\delta$-импульс падает на записанную структуру с правой стороны.

Известны голографические методы восстановления временной структуры коротких оптических импульсов [31,32]. Мы полагаем, что в нашем примере мы имеем дело с другим механизмом восстановления. Возможно, он близок к механизму формирования временной структуры светового импульса брэгговской решеткой [33], только здесь мы оперируем в пространственновременно́й области вместо спектральной.

\section{Заключение}

Продемонстрировано отличие ГО механизма восстановления волнового фронта от голографического. ГО восстановление волнового фронта было получено при использовании ультракоротких лазерных импульсов при записи в объемной регистрирующей среде и в планарном волноводе. Предложено несколько вариантов ахроматических ОЭ и оптических систем. Предложен оптикоакустический фокусирующий элемент, способный создавать большие локальные напряжения в области заданной формы внутри среды. Продемонстрирована возможность восстановления временной структуры оптических импульсов с помощью ГО отражения.

\section{Финансирование работы}

Работа выполнена в рамках государственного задания.

\section{Конфликт интересов}

Автор заявляет, что у него нет конфликта интересов.

\section{Список литературы}

[1] Денисюк Ю.Н. // Опт. и спектр. 1963. Т. 15. № 4. С. 522; Denisyuk Yu.N. // Opt. Spectrosc. (USSR) 1963. V. 15. P. 279.

[2] Сисакян И.Н., Смолович А.М. // Письма в ЖТФ. 1991. T. 17. № 1. C. 41; Sisakyan I.N., Smolovich A.M. // Sov. Tech. Phys. Lett. 1991. V. 17. P. 16.

[3] Smolovich A.M. // Appl. Optics. 2006. V. 45. N 30. P. 7871.

[4] Кравцов Ю.А., Орлов Ю.И. Геометрическая оптика неоднородных сред. М.: Наука, 1980. 304 с.; Kravtsov Yи.A., Orlov Yu.I. Geometrical Optics of Inhomogeneous Media. Springer-Verlag, 1990.

[5] Gabor D. // Proc. R. Soc. Lond. A. 1949. V. 197. N 1051. P. 454.

[6] Борн М., Воль $б$ Э. Основы оптики. М.: Наука, 1970. 856 с.; Born M., Wolf E. Principles of Optics. Cambridge: Cambridge Univ. Press, 2002. doi 10.1017/CBO9781139644181

[7] Meier R.W. // JOSA. 1965. V. 55. N 8. P. 987.

[8] Сисакян И.Н., Смолович А.М. // Компьютерная оптика. 1990. № 7. С. 56.

[9] Chekalin S.V., Dement'ev D.A., Ivanov A.L., Matveets Yu.A., Serov O.B., Smolovich A.M., Stepanov A.G. // Proc. SPIE. 1999. V. 3733. P. 452.

[10] Дементьев Д.А., Иванов А.Л., Серов О.Б., Смолович А.М., Степанов А.Г., Чекалин С.В. // Письма в ЖЭТФ. 1997. T. 65. № 5. C. 388; Dement'ev D.A., Ivanov A.L., Serov O.B., Smolovich A.M., Stepanov A.G., Chekalin S.V. // JETP Lett. 1997. V. 65. P. 402.

[11] Chekalin S.V., Dement'ev D.A., Ivanov A.L., Matveets Yu.A., Serov O.B., Smolovich A.M., Stepanov A.G. // Optics Commun. 1998. V. 150. N 1-6. P. 38.

[12] Gabor D., Stroke G.W. // Proc. R. Soc. Lond. A. 1968. V. 304. N 1478. P. 275.

[13] Дементьев Д.А., Матвееи, Ю.А., Серов О.Б., Смолович А.М., Чекалин С.В. // Квант. электрон. 1996. Т. 23. № 4. C. 293; Dement'ev D.A., Matveets Yu.A., Serov O.B., Smolovich A.M., Chekalin S.V. // Quant. Electron. 1996. V. 26. P. 285.

[14] Любавская И.К., Серов О.Б., Смолович А.М. // Письма в ЖТФ. 1980. Т. 6. № 1. C. 38; Lyubavskaya I.K., Serov O.B., Smolovich A.M. // Sov. Tech. Phys. Lett. 1980. V. 6. P. 16.

[15] Smolovich A.M., Álvarez E., Aseyev S.A., Cervantes M.A., Chekalin S.V., Serov O.B., Terpugov V.S. // Optics Lett. 2008. V. 33. N 20. P. 2401.

[16] Smolovich A.M., Álvarez E., Aseyev S.A., Cervantes M.A., Chekalin S.V., Irzhak A.V., Serov O.B., Terpugov V.S. // Proc. SPIE. 2007. V. 6732. P. 6732-12.

[17] Stetson K.A. // Laser Focus. 1967. V. 3. N 5. P. 25.

[18] Sheridon N.K. // Appl. Phys. Lett. 1968. V. 12. N 9. P. 316.

[19] Lesem L.B., Hirsch P.M., Jordan J.A. // IBM J. Research and Development. 1969. V. 13. N 2. P. 150.

[20] Kovatchev M., Ilieva R. Inphase optical processors. 1. Inphase structures in optical computing // Invited paper 12A1, in Proceedings of Optical Computing. Kobe, Japan. 1990. V. 90. P. 389.

[21] Faklis D., Morris G.M. // Appl. Opt. 1995. V. 34. N 14. P. 2462. 
[22] Sweeney D.W., Sommargen G.E. // Appl. Opt. 1995. V. 34. N 14. P. 2469.

[23] Rossi M., Kunz R.E., Herzig H.P. // Appl. Opt. 1995. V. 34. N 26. P. 5996.

[24] Грейсух Г.И., Данилов В.А., Степанов С.А., Антонов А.И., Усиевич Б.А // Опт. и спектр. 2018. Т. 125. № 2. C. 223. doi 10.21883/OS.2018.08.46364.96-18; Greisukh G.I., Danilov V.A., Stepanov S.A., Antonov A.I., Usievich B.A. // Opt. Spectrosc. 2018. V. 125. N 2. P. 232.

[25] Сисакян И.Н., Смолович А.М., Сойфер В.А. Патент РФ № 1620973, 1991.

[26] Сисакян И.Н., Смолович А.М., Сойбер В.А. // Компьютерная оптика. 1989. № 6. С. 3.

[27] Аскарьян Г.А., Гольдштейн Р.В., Капщов А.В., Сисакян И.Н., Смолович А.М., Юркин А.В. Патент РФ № $1749250,1992$.

[28] Аскарьян Г.А., Клебанов Л.Д. // Квант. электрон. 1988. T. 15. № 11. C. 2167.

[29] Аскарьян Г.А., Королев М.Г., Юркин А.В. // Письма в ЖЭТФ. 1990. Т. 51. № 11. С. 586.

[30] Cervantes M.A., Smolovich A.M. // Proc. SPIE. 2002. V. 4752. P. 66.

[31] Rebane A., Kaarli R., Saari P., Anijalg A., Timpmann K. // Opt. Commun. 1983. V. 47. N 3. P. 173.

[32] Mazurenko Y.T. // Appl. Phys. B. 1990. V. 50. N 2. P. 101.

[33] Hill K.B., Brady D.J. // Opt. Lett. 1993. V. 18. N 20. P. 1739. 